\title{
A NEW SURVEY ON SELF-TUNING INTEGRATED LOW-COST GPS/INS VEHICLE NAVIGATION SYSTEM IN HARSH ENVIRONMENT
}

\author{
Néda Navidi and René Jr Landry \\ Département de génie électrique \\ École de technologie supérieure (ETS), Montréal, Canada \\ Neda.navidi@lassena.etsmtl.ca; ReneJr.landry@etsmtl.ca
}

KEY WORDS: GPS/INS integration, Extended Kalman Filter, Fuzzy Inference System

\begin{abstract}
:
Nowadays, Global Positioning System (GPS) receivers are aided by some complementary radio navigation systems and Inertial Navigation Systems (INS) to obtain more accuracy and robustness in land vehicular navigation. Extended Kalman Filter (EKF) is an acceptable conventional method to estimate the position, the velocity, and the attitude of the navigation system when INS measurements are fused with GPS data. However, the usage of the low-cost Inertial Measurement Units (IMUs) based on the MicroElectro-Mechanical Systems (MEMS), for the land navigation systems, reduces the precision and stability of the navigation system due to their inherent errors. The main goal of this paper is to provide a new model for fusing low-cost IMU and GPS measurements. The proposed model is based on EKF aided by Fuzzy Inference Systems (FIS) as a promising method to solve the mentioned problems. This model considers the parameters of the measurement noise to adjust the measurement and noise process covariance. The simulation results show the efficiency of the proposed method to reduce the navigation system errors compared with EKF.
\end{abstract}

\section{INTRODUCTION}

Accuracy requirement of Location-Based Telematics Services (LBTS) for land navigation mostly depends on Global Positioning System (GPS) and Global Navigation Satellite Systems (GNSS) to obtain a useful navigational solution. GNSS receivers require proper operating conditions for providing a valid and precise solution. Whereas GNSS operation is affected by Line of Sight (LOS) signal propagation and condition, resulting in a non-effective GNSS solution while driving in harsh environments, such as in urban canyons, under dense foliage or other so-called GPSdenied environment (i.e. indoor parking, tunnel, etc.). As a matter of fact, these harsh environments significantly reduce the efficiency of GNSS [1,2].

Advanced commercial vehicle services, such as car insurance companies and car assistance services (OnStar, Car-net, etc.), need to overcome the mentioned GNSS problems to provide higher customer supports and quality of services. An encouraging solution to achieve a high-precision navigation system, at all time, is to fuse the GPS data with low-cost Micro-Electro-Mechanical Systems (MEMS)-based Inertial Measurement Units (IMUs), which are self-governing navigation devices. In fact, an GPS/INS integrated system can provide a state-of-the-art land navigation system with a better efficiency compared with GPS or INS alone.

The conventional Extended Kalman Filter (EKF) has been extensively employed for the integration of INS with GPS, as it can determine the optimal estimation of the system state vector with minimum mean and square errors [3-6]. For good results, an EKF needs to perform under appropriate defined errors and dynamic models notably for its initialization process, which requires the knowledge and adequate noise modeling. Also and to provide a continuous efficient estimation, EKF requires a good background on the dynamic process of the system and measurement model based on white Gaussian noises. In addition, the accuracy of MEMSbased IMUs is greatly damaged by their long and short terms drift and bias errors. This problem impacts the navigation systems' performance, when exploiting the traditional EKF in land vehicular navigation [7]. Moreover, the main problem of the EKF is the covariance divergence due to modeling error in EKF, which makes infinite or very large the bound of the actual estimate error covariance [8].

To solve the covariance divergence in EKF, two solutions are mainly considered. The first one is employing the unmodeled state which increases the complexity of the system for its computation [8]. The second is employing the process noise to improve the confidence when using white Gaussian noise so that it can prevent the EKF to reject new measurements for estimating the state vector $[7,9,10]$. This paper suggests using Fuzzy Inference System (FIS) based on the second solution, which is employed for self-tuning the EKF parameters by observing the covariance measurements.

The proposed hybrid FIS-EKF model is exploited to develop a low-cost and low-processing power GPS/INS integrated navigation system for modern land vehicular navigation systems efficient in challenging environments. This new technique will open the door to new navigation capabilities to expand the markets of automotive navigation services. FIS is exploited to predict the error states of the EKF based on a Covariance Matching Estimation Technique (COMET). The paper is organized as follows: Section 2 presents the GPS/INS integration process used in this paper. Section 3 explains the proposed model to fuse the INS and GPS measurements in detail. Sections 4 and 5 present the simulation results and the conclusion of this paper, respectively.

\section{GPS/INS INTEGRATION PROCESS}

\section{A. Loosely coupled GPS/INS integration}

It is well known that integration of INS and GPS through loosely coupled structure, not only maintains independency and redundancy of stand-alone GPS and INS solutions; but it can also provide more robustness for the navigation solution [11]. Loosely coupled integration presents a closed-loop 
architecture that allows the correction of certain errors of the INS. This structure is generally preferable in the GPS/INS integration as being composed of three distinct entities, which are the stand-alone GPS solution, the stand-alone INS solution and the GPS/INS coupled solution. This architecture, presented in Figure 1, is shared by several authors [12-14]. In this architecture, a filter is used to fuse the GPS and INS solutions (position and velocity) and to estimate some system error's parameters. The estimated parameters correspond to the errors of position, velocity, and attitude of the INS solution.

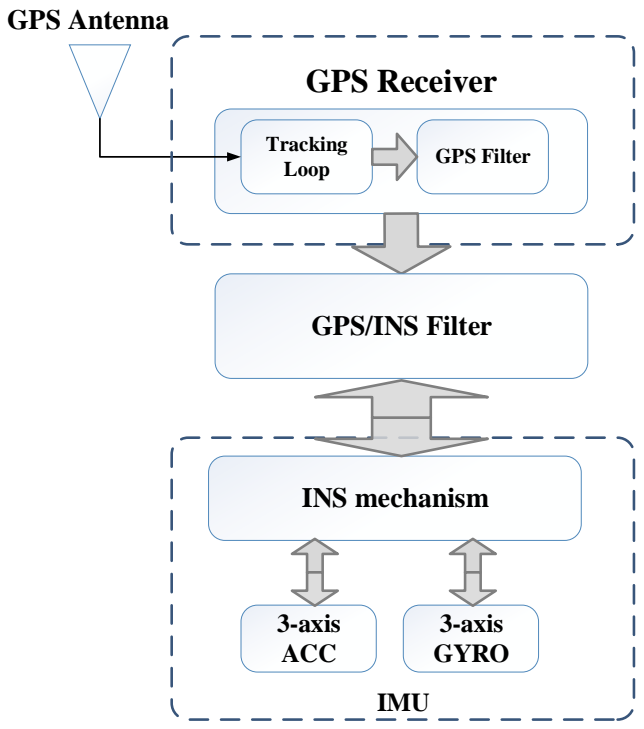

Figure 1. Loosely architecture of GPS/INS integration

In general, the filters in Figure 1 are EKFs; which are recognized as the most conventional method to estimate the navigation systems. The derivation of the error model to be applied in the EKF starts with the construction of the full scale true error models, whose order is decided from the complexity of the problem [15]. The error model in the dynamical model includes three state errors of position, three for the velocity, and three for the attitude, augmented by some sensor state errors. GPS/INS integration reduces only the long-term errors with this error model. Thus, the remaining error budget is mainly affected by short-term error sources [16].

\section{B. Extended Kalman Filters (EKF) process}

The EKF is an estimator method, which can distinguish the problem of estimating the state of a controlled process. It is defined by the following linear dynamic model:

$$
x_{k}=F_{k-1} x_{k-1}+G_{k-1} w_{k-1}
$$

with a measurement that is:

$$
z_{k}=H_{k} x_{k}+v_{k}
$$

where $F_{k}, G_{k}$ and $H_{k}$ are the state transition, the system noise coefficient and the design matrices, respectively, $x_{k}$ is the error state vector, and $z_{k}$ is the measurement vector. The random variables $w_{k}$ and $v_{k}$ represent the system and measurement noises, respectively, with uncorrelated and zero mean random processes:

$$
\begin{gathered}
E\left[w_{k} v_{j}^{T}\right]=0 \quad \forall i, j \in \mathbb{R} \\
w_{k} \sim \mathcal{N}\left(0, Q_{k}\right) \\
v_{k} \sim \mathcal{N}\left(0, R_{k}\right)
\end{gathered}
$$

and the covariance matrices are:

$$
\begin{aligned}
& E\left[w_{k} w_{j}^{T}\right]=\left\{\begin{array}{cc}
Q_{k} & , j=k \\
0 & , j \neq k
\end{array}\right. \\
& E\left[v_{k} v_{j}^{T}\right]=\left\{\begin{array}{cc}
R_{k} & , j=k \\
0 & , j \neq k
\end{array}\right.
\end{aligned}
$$

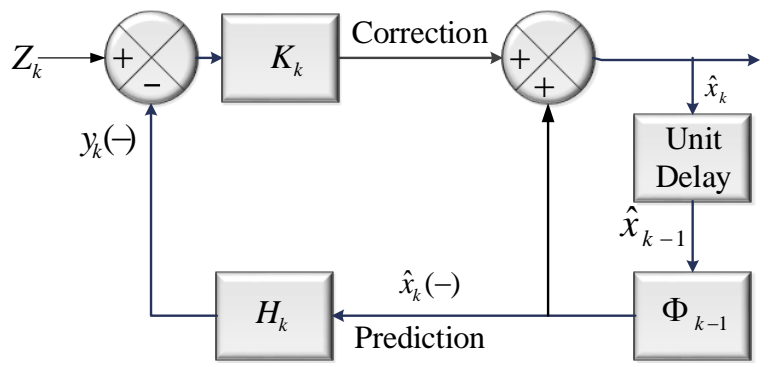

Figure 2. A block diagram of Extended Kalman Filter

In reality, the system noise covariance $Q_{k}$, and measurement noise covariance $R_{k}$ matrices might change with each time step. However, they are often assumed to be constant in EKF to facilitate the implementation [15]. There are two steps in EKF process. A block diagram of EKF is shown in Figure 2. The first step is the prediction of the system model:

$$
\begin{gathered}
\hat{x}_{\bar{k}}=F_{k-1} \hat{x}_{k-1} \\
P_{\bar{k}}=F_{k, k-1} P_{k-1} F_{k-1}^{T}+G_{k-1} Q_{k-1} G_{k-1}^{T}
\end{gathered}
$$

where, $\hat{x}_{\bar{k}}$ and $P_{\bar{k}}$ are the time-propagated state estimates and covariance. The second step is the measurement update of the system model:

$$
\begin{array}{lc}
\text { Kalman gain: } & K_{k}=P_{\bar{k}} H_{k}^{T}\left(H_{k} P_{\bar{k}} H_{k}^{T}+R_{k}\right)^{-1} \\
\text { Covariance } & P_{k}=\left(I-K_{k} H_{k}\right) P_{\bar{k}} \\
\text { update: } & \hat{x}_{k}=\hat{x}_{\bar{k}}+K_{k}\left(z_{k}-H \hat{x}_{\bar{k}}\right)
\end{array}
$$

\section{Fuzzy Inference Systems (FISs)}

Fuzzy Inference Systems (FISs) is a rule-based expert method for its ability to mimic human thinking and the linguistic concepts rather than the typical logic systems [17]. The advantage of the FIS appears when the algorithm of the estimation states become unstable due to the system high complexity. FIS are also used for the knowledge induction process as they are applicable estimators for general purpose [18].

A FIS architecture includes three parts: fuzzification, fuzzy inference and defuzzification. The first part is responsible to convert the crisps input values to the fuzzy values, the second part formulates the mapping from the given inputs to an output, and the third part converts the fuzzy operation into the new crisp values. The FIS are able to convert the inaccurate data to normalized fuzzy crisps which are represented by the ranges of possible values, Membership Functions (MF) and the confidence-rate of the inputs. In addition, the FIS are capable to choose an optimal MF under certain convenient criteria meaningful to a specific application. The deterministic output of FIS and its 
performance depend on the effective fuzzy rules, the considered defuzzification process and the reliability of the MF values $[19,20]$.

In Fuzzy theory, components are referred to a range of values between 0 and 1 . It is in contrast to crisp sets where a component is either in a set or not in the set. The range of these values expresses the MF components in the FIS. Mamdani and Sugeno are the two practical FIS types which are used in several studies [21-24]. The main difference between these two fuzzy algorithms is based on the process complexity and the rule definition. Another important aspect to take into consideration is that Mamdani needs more processing time than Sugeno. In low signal to noise (SNR) scenarios, Mamdani type acts more robustly compared to Sugeno. Sugeno type provides also less flexibility in the system design compared to the Mamdani type. In general, Mamdani type is more efficient and accurate than Sugeno type $[21,22]$. All those reasons have motivated us to use the Mamdani type (see Figure 3) to design the fuzzy-part of the proposed GPS/INS integration model.

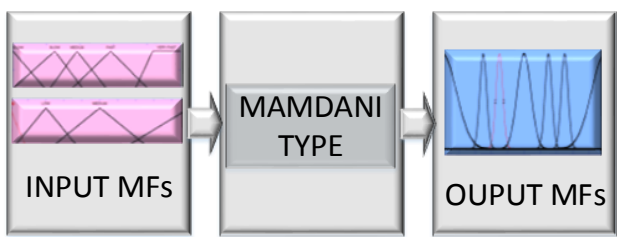

Figure 3. General overview of FIS using Mamdani type

\section{PROPOSED ADAPTIVE FIS-EKF SYSTEM}

Two different approaches of adaptive Kalman filtering, namely Innovation Adaptive Estimation (IAE) and Multiple Model Adaptive Estimation (MMAE), are considered by several research groups $[20,25,26]$. These two approaches are based on COMET, which uses updated statistical data. This paper employs the IAE concept in the fuzzy part of the proposed model.

The dynamic characteristics of the vehicle motion in the body frame to the navigation frame are the bases of the EKF process. The FIS can be exploited to increase the accuracy and the robustness of the EKF as well as preventing its divergence, in the tuning phase of EKF, especially in poorly designed geometric and mathematical models. Because, these models in the EKF, may lead to the divergence. Hence, FIS is used as a structure for identifying the dynamical variations and implementing the real-time tuning of the nonlinear error model. It can provide a good estimation to maintain the accuracy and the tracking-capability of system. Figure 4 depicts how the proposed hybrid FIS-EKF model performs as the ubiquitous navigation system.

The proposed FIS model is based on the IAE and innovation process of the covariance matrix for the input of the FIS as well as the difference of the actual covariance matrix and the estimated covariance matrix. Figure 5 shows the proposed FIS overview that is used in this paper. The estimated covariance matrix based on the innovation process is computed partly in the EKF by:

$$
\hat{S}_{k}=H_{k} P_{\bar{k}} H_{k}^{T}+R_{k}
$$

where $\hat{S}_{k}$ and $H_{k}$ represent the estimated covariance and the design matrices, respectively. $P_{\bar{k}}$ and $R_{k}$ are previous covariance update and the measurement noise covariance matrices. The actual covariance matrix $(\gamma)$ according to [27] can be presented by:

$$
\gamma_{k}=\frac{1}{W} \sum_{i=k-W+1}^{k}\left(z_{i}-H_{i} \hat{x}_{\bar{l}}\right) \times\left(z_{i}-H_{i} \hat{x}_{\bar{l}}\right)^{T}
$$

where $W$ is the window size which is given by the moving window technique. It is chosen experimentally in this paper as $W=25$. So, the difference between the actual and estimated covariance matrices $(\varepsilon)$ can be presented by:

$$
\begin{aligned}
\varepsilon_{k} & =\gamma_{k}-\hat{S}_{k} \\
& =\frac{1}{W} \sum_{i=k-W+1}^{k}\left(z_{i}-H_{i} \hat{x}_{\bar{l}}\right) \times\left(z_{i}-H_{i} \hat{x}_{\bar{l}}\right)^{T} \\
& \quad-H_{k} P_{\bar{k}} H_{k}^{T}+R_{k}
\end{aligned}
$$

In fact, the value of $\varepsilon$ can display the level of divergence between the actual and the estimated covariance matrices. This value depicts the level of the alteration in the vehicle dynamic based on the related covariance matrices. When the value of $\varepsilon$ is close to zero, the estimated and the actual covariance matrices will be very similar and the absolute value of the $\varepsilon$ can be neglected. However, if the value of $\varepsilon$ is not near to zero (smaller or greater than zero), an adaptation is considered in the algorithm and the value of $R_{k}$ in Equation (11) should be adjusted to compensate this difference.

The proposed rules assessment according to the difference between the actual and the estimated covariance matrices is described as three scenarios of MF:

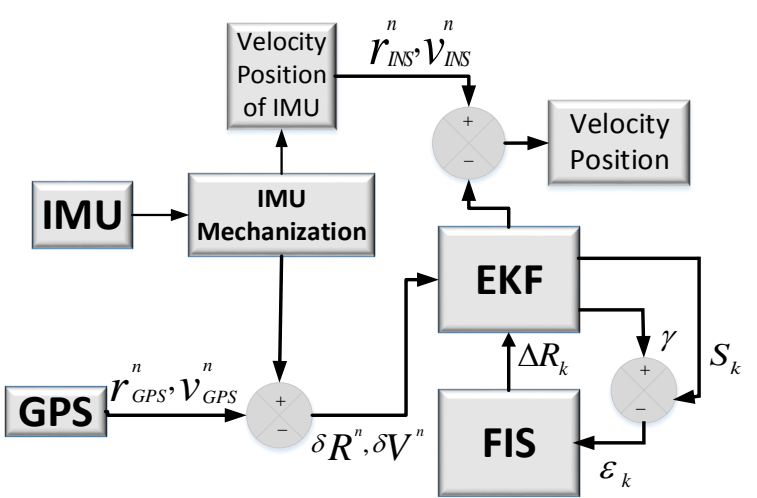

Figure 4. The proposed hybrid FIS-EKF model in navigation system

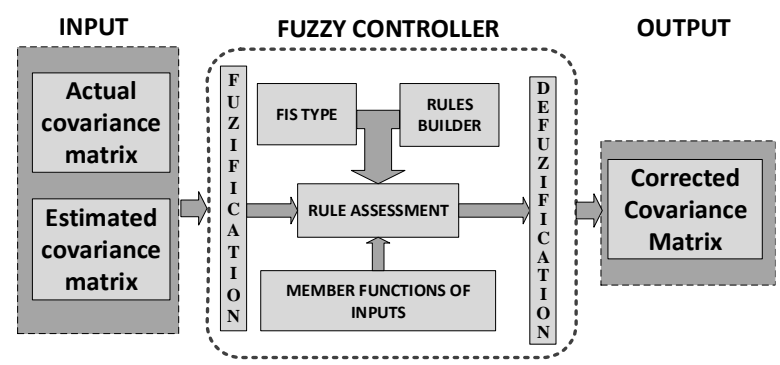

Figure 5. The proposed FIS part of the hybrid FIS-EKF in navigation system. 
1) If the value of $\varepsilon$ is higher than zero, then the value of $R_{k}$ is turned down in accordance with the value of $\delta \mathrm{R}_{k}$;

2) If the value of the $\varepsilon$ is less than zero, then the value of $\mathrm{R}_{k}$ is turned up in accordance with the value of $\delta \mathrm{R}_{k}$;

3 ) If the value of $\varepsilon$ is close to zero, then $\mathrm{R}_{k}$ is unchanged.

where, $\delta \mathrm{R}_{k}=\mathrm{R}_{k}-\mathrm{R}_{k-1}$. Before using the value of $\delta \mathrm{R}_{k}$ in the fuzzy controller, they should be fuzzified in the linguistic term using the related MF. The parameter $\delta \mathrm{R}_{k}$ in Figure 6(a) is represented by the five linguistic terms, namely ' $\mathrm{I} 1$ ', ' $\mathrm{I} 2$ ', 'I3', 'I4', and 'I5',

Figure 6(a) explains the grade of the membership parameter in the five fuzzy sets. For example, if $\delta \mathrm{R}_{k}$ is close to zero, the grade of MF is shown with I2 in the FIS. As the degree of MF in the fuzzy set is I1 or I4, it approaches to minus one. However, when parameter of $\delta \mathrm{R}_{k}$ is one, there is a progressive transition from I 2 to $\mathrm{I} 3$ or $\mathrm{I} 5$, which is performed by the overlapping period in Figure 6(a). Then, the different values of this period are referred to both fuzzy sets with various grades of membership functions.

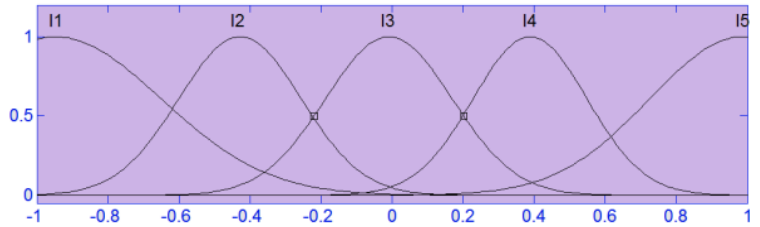

(a)

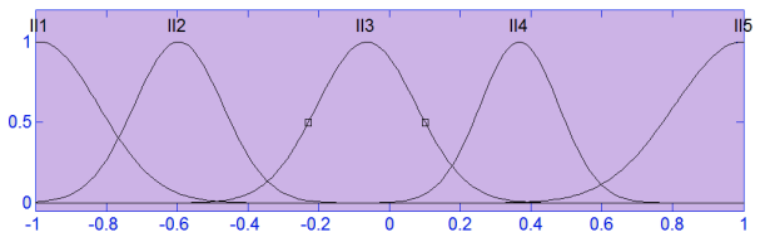

(b)

Figure 6. Membership Functions (MF) of inputs: (a) Value of $\varepsilon$ (b) Value of $\delta \varepsilon$.

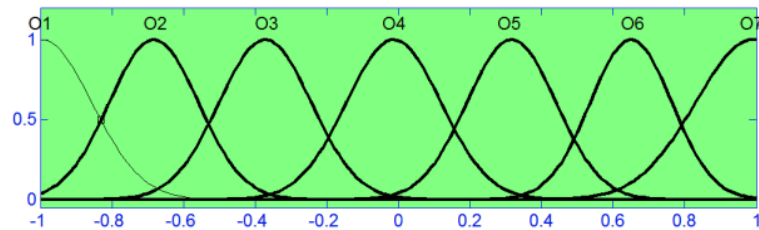

Figure 7. Membership Functions (MF) of output $\left(\delta \mathrm{R}_{k}\right)$ for proposed FIS

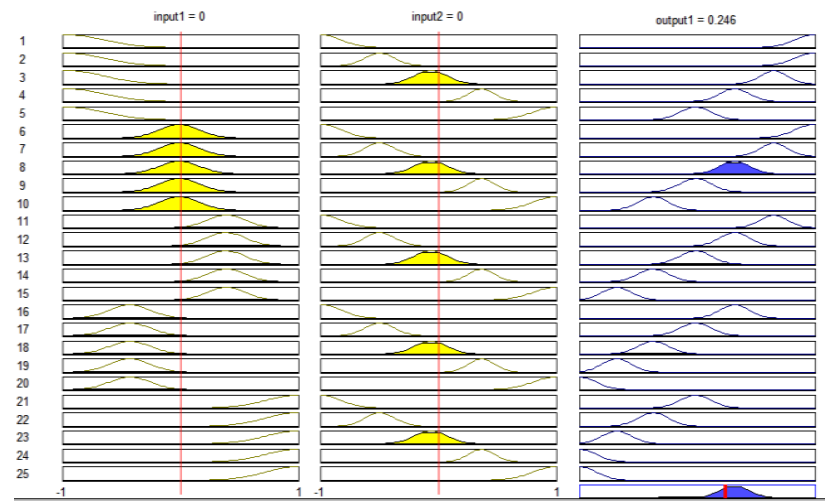

Figure 8. The rules builder in proposed FIS-part
In addition, after rewriting Equation (11) using Equation (7), we have:

$$
\begin{gathered}
\hat{S}_{k}=H_{k}\left(F_{k, k-1} P_{k-1} F_{k, k-1}{ }^{T}+G_{k-1} Q_{k-1} G_{k-1}{ }^{T}\right) H_{k}^{T} \\
+R_{k}
\end{gathered}
$$

which shows that if the $\mathrm{R}_{k}$ is perfectly observed, variation in $Q_{k}$ can make a change in $\hat{S}_{k}$ directly. So, with the observation of the mismatch between the actual and the estimated covariance matrices, an augmentation or reduction is essential for $Q_{k}$. The proposed rules assessment for this purpose is described as two MF of FIS:

1) If $\varepsilon_{k}-\varepsilon_{k-1}=0$, then $Q_{k}$ will be unchanged;

2) If $\varepsilon_{k}-\varepsilon_{k-1} \neq 0$, then $Q_{k}$ will be changed in accordance with the value of $\delta Q_{k}$.

where $\delta Q_{k}=Q_{k}-Q_{k-1}$. The related MF are shown in Figure 6(b) with 'II1','II2', 'II3','II4' and 'II5'. 'II1' and 'II2' are used when the value of $\varepsilon_{k}$ is lower than zero. II3 is employed when the value of $\varepsilon_{k}$ is zero. II4 and II5 are considered as the value of $\varepsilon_{k}$ is greater than zero.

The MF of the output for the proposed FIS model in order to match the covariance matrix is presented in the Figure 7. The output is the value of $\delta \mathrm{R}_{k}$ which changes from -1 to 1 . Moreover, it is fuzzified in seven levels from $\mathrm{O} 1$ to $\mathrm{O} 7$. These seven levels are important to differentiate between the likelihood levels. Figure 8 depicts the rules builder of the proposed FIS part. In fact, the rules builder defines the relationships between the two inputs and the outputs of the proposed model. Figure 9 presents the result of the rules builder in three dimensions. This figure shows that a change of the values of $\varepsilon$ and $\delta \varepsilon$ inputs affects the value of the output $\delta \mathrm{R}_{k}$. The next section presents the performance of the proposed FIS/EKF within simulation experiments.

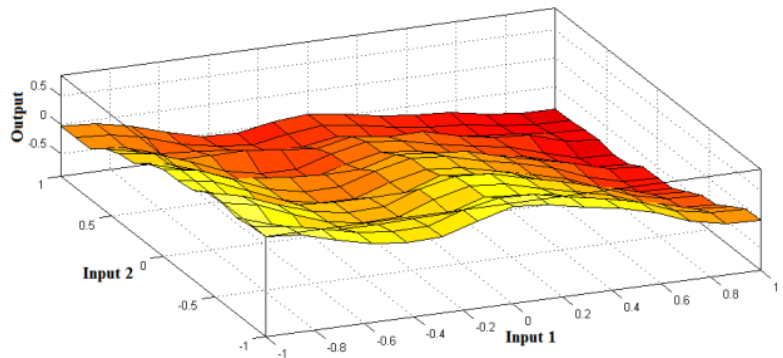

Figure 9. Variation of $\boldsymbol{\delta} R_{\boldsymbol{k}}$ (Output) based on the change of the values of $\varepsilon$ and $\delta \varepsilon$ (Inputs 1 and 2)

\section{SIMULATION EXPERIMENTS AND ANALYSIS}

To evaluate the performance of the proposed GPS/INS integration model, simulation results of the FIS/EKF method are compared with those obtained from conventional GPS/INS integration method. For the demonstration of the proposed model, a loosely coupled GPS/INS integration in two-dimension is considered to reduce the overall cost and complexity of the navigation system (see Figure 10). The error state related to the two-dimensional inertial navigation system in this paper can be presented as:

$$
\begin{aligned}
& \delta x=\left[\begin{array}{lllll}
\delta n & \delta e & \delta v_{n} & \delta v_{e} & \delta \psi
\end{array}\right]^{T} \\
& \delta x=\left[\begin{array}{c}
v_{n} \\
v_{e} \\
\operatorname{Cos}(\psi) a_{x}-\operatorname{Sin}(\psi) a_{y} \\
\operatorname{Sin}(\psi) a_{x}+\operatorname{Cos}(\psi) a_{y} \\
\psi_{r}
\end{array}\right]
\end{aligned}
$$


where $\delta n$ and $\delta e$ are the north and the east position errors, and $\delta v_{n}$ and $\delta v_{e}$ are the north and the east velocity errors, respectively. $\delta \psi$ represents the heading error. $\left[\begin{array}{ll}a_{x} & a_{y}\end{array}\right]$ and $\psi_{r}$ show the measured accelerations and yaw-rate in body frame.

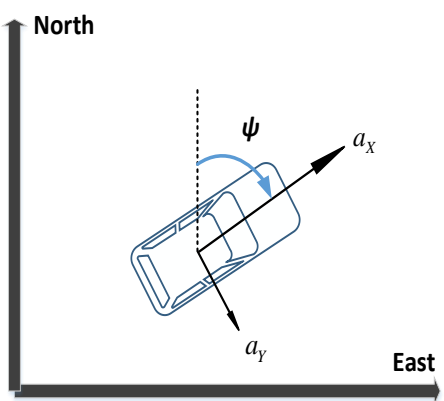

Figure 10. Two-dimensional navigation system

The vehicle trajectory was generated using the MATLAB Software Satellite Navigation toolbox in different time intervals and motions, which are presented in Figure 11. The initial location is defined as $(0,0) \mathrm{m}$ in local frame. Then three main time intervals are considered for the straight and circle motions of the trajectory. The details of the trajectory are described in Table 1 . The vehicle is simulated to drive with a constant speed of $4.5 \pi \mathrm{m} / \mathrm{s}$.

Table 1. Definition of the trajectory

\begin{tabular}{|c|c|}
\hline Time segment (s) & Motion \\
\hline$[0-1200]$ & Straight \\
\hline$[1201-2400]$ & counter-clockwise circle \\
\hline$[2401-3600]$ & Straight \\
\hline
\end{tabular}

Tree main points are considered for GPS availability signals in the defined scenario. Fist, satellite positions are simulated without different errors; because it is needed a perfect reference to validate the proposed FIS/EKF within simulation experiments. It is called 'Perfect situation of GPS' in this paper. Second, satellite constellations are generated in the present of different error sources like multipath, clock errors, atmospheric errors and receiver noise. Most of these error sources are neglected, while, multipath and receiver noise cannot be disregarded in second scenario. It is called 'Normal situation of GPS' that maximum GPS unavailability is five seconds. Third, during the trajectory, ten obstructed segments were considered and introduced in GPS signals. It is called 'bad situation of GPS' which are shown with the circle pink numbers in Figure 11. Table 2 shows the defined duration, start point and stop point of each GPS signal outage (it is assumed that right after this duration, the GPS position is again available). Minimum and maximum defined durations are $48 \mathrm{~s}$ and $97 \mathrm{~s}$. This is used to demonstrate the system performance in severe environment (urban canyons, overpasses, tunnels, and indoor parking). The update rate of the GPS for all situations is one second.

There are different stochastic errors in INS, which are modeled by different error states like biases and drifts of accelerometers and gyroscopes. However, low-cost INSs augmented by the modeled noises, cannot consistently perform as a ubiquitous navigator; especially in long GPS absence. Therefore, a model-free method can solve the problem of noise modeling in low-cost INSs. The assumed INS errors in this paper are summarized in Table 3.

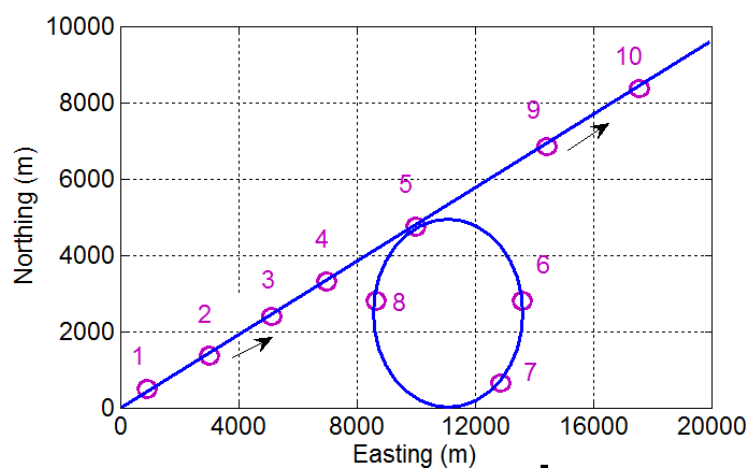

Figure 11. Trajectory in three main time-segments due to the dynamics characteristic.

Table 2. Definition of the GPS outages based on their durations in the trajectory.

\begin{tabular}{|c|c|c|c|}
\hline Outages (\#) & Period (s) & Start point (s) & Stop point (s) \\
\hline 1 & 62 & 17 & 79 \\
\hline 2 & 60 & 296 & 356 \\
\hline 3 & 88 & 590 & 678 \\
\hline 4 & 90 & 896 & 986 \\
\hline 5 & 88 & 1,202 & 1290 \\
\hline 6 & 92 & 1,510 & 1602 \\
\hline 7 & 97 & 1,795 & 1892 \\
\hline 8 & 83 & 2,160 & 2243 \\
\hline 9 & 68 & 2,810 & 2878 \\
\hline 10 & 48 & 3,230 & 3278 \\
\hline
\end{tabular}

Table 3. Definition of the INS errors

\begin{tabular}{|c|c|}
\hline Parameters & Value \\
\hline$A c c_{x}$-bias & $8 \mathrm{~g}$ \\
\hline$A c c_{y}$-bias & $8 \mathrm{~g}$ \\
\hline Gyr-bias & $0.15 \mathrm{deg} / \mathrm{s} ;$ \\
\hline Acc-noise & $0.1 \mathrm{~m} / \mathrm{s}^{2}$ \\
\hline Gyr-noise & $0.1 \% / \mathrm{s}^{2}$ \\
\hline
\end{tabular}

Figure 12 shows the results in normal situation of GPS where there is no GPS outage more than five seconds. Figure 14 (a) presents a comparison of Root Mean Square Errors (RMSE) between EKF and the proposed hybrid FIS-EKF model in normal situation of GPS. As it is shown in this figure, the proposed model attenuates the errors by $10 \%$ and $11.28 \%$ in the east and the north position, respectively, compared to the traditional EKF when the GPS is working in normal situation. Also, the usage of the FIS-EKF reduces the east and the north velocity errors as well as the heading error by approximately $16.2 \%, 27.9 \%$ and $12 \%$, respectively. Figure 13 depicts the errors of the conventional EKF and proposed hybrid FIS-EKF model during the ten assumed outages. Figure 14 (b) presents a comparison of RMSEs between EKF and the proposed hybrid FIS-EKF model.

This figure obviously illustrates the significant improvement of navigation solution when using the proposed FIS-EKF model. It is observed that, after applying FIS-EKF, errors were decreased by $48.48 \%$ and $49.72 \%$ in the east and north positions compare to EKF. Furthermore, the proposed model presents the enhancement in the navigation performance for the east and the north velocity as well as the heading by $51.56 \%, 49.02 \%$ and $72.91 \%$, respectively. 


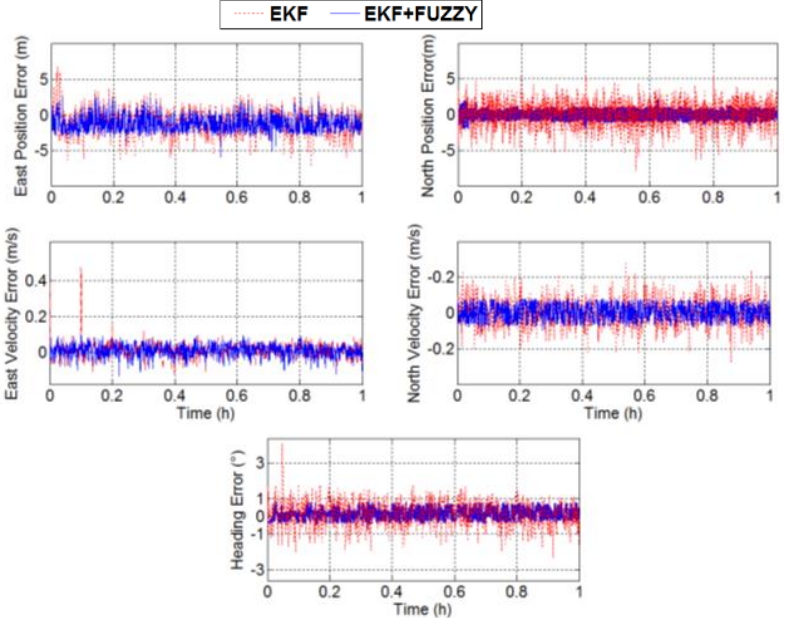

Figure 12. Position, velocity, and heading errors in normal situation of GPS
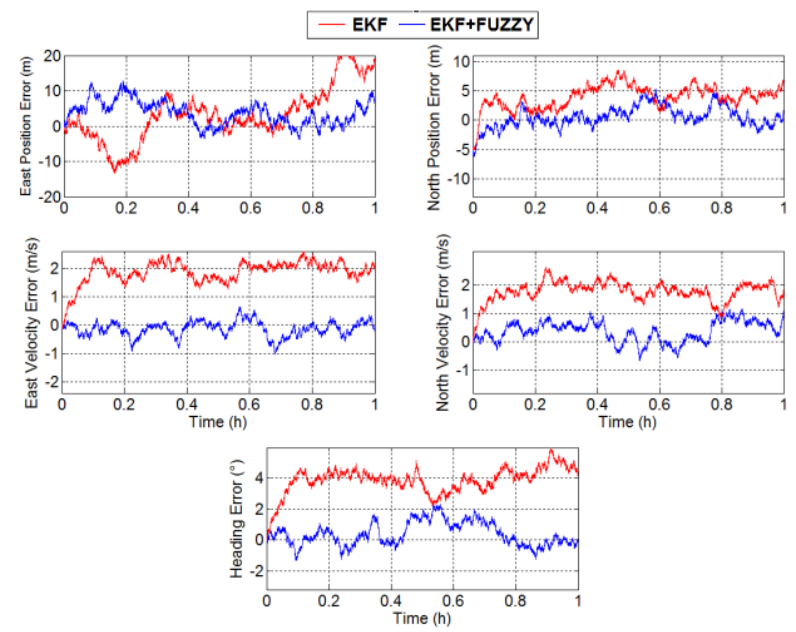

Figure 13. Position, velocity, and heading errors during the bad situation of GPS

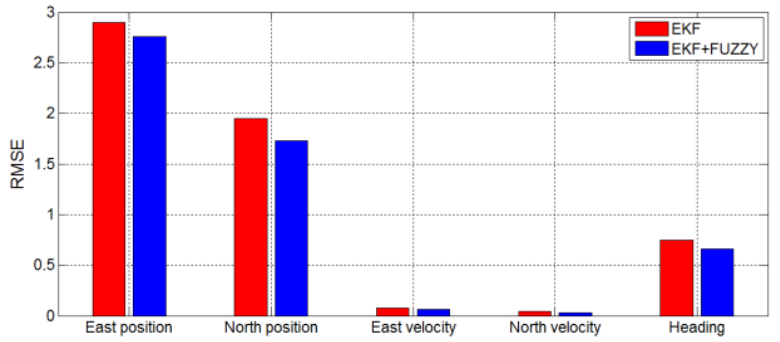

(a)

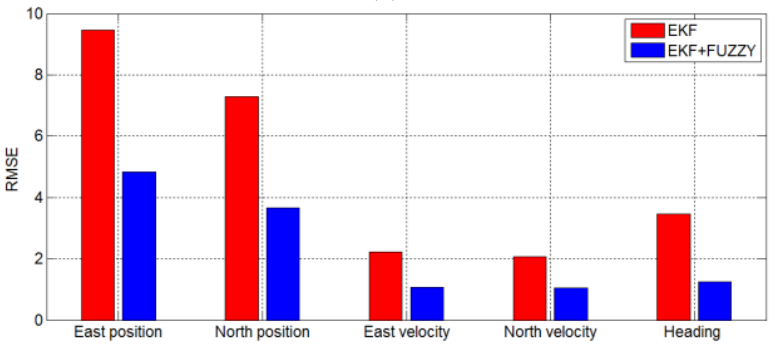

(b)

Figure 14. Comparison of position and velocity RMS errors via two approaches: EKF and FUZZY-EKF (a) Normal situation of GPS; (b) Bad situation of GPS

\section{CONCLUSION}

This paper has proposed the improvement of standard loosely coupled GPS/INS integration based on a hybrid EKF and a fuzzy inference system. The proposed model improves the performance of the navigation system, whereas the traditional EKF needs a priori knowledge of the system. As shown by the simulation experiments, the traditional EKF does not perform well in GPS outages, especially using low-cost IMU. Thus, the proposed FIS-EKF is exploited to increase the accuracy and the robustness of the EKF as well as to prevent the divergence in the EKF tuning process due to poorly design of the geometric models. The proposed FIS model is based on the IAE and innovation process of the covariance matrix as the input of the FIS, as well as the difference of the actual covariance matrix and the estimated covariance matrix. The major benefit of the proposed model is that the positioning accuracy of this model is increased compared to usual EKF-based integration. The main reason is that the estimated value of the covariance matrix is adapted to its actual value in the proposed hybrid FIS-EKF model. The results confirmed that higher accuracy is achieved using proposed hybrid FIS-EKF model compared to EKF-alone. The next work will consist to verify it viability with real GPS and IMU measurements acquired in real harsh environment.

\section{ACKNOWLEDGEMENTS}

This research is part of the project entitled "VTADS: Vehicle Tracking and Accident Diagnostic System". This research is partially supported by the Natural Sciences and Engineering Research Council of Canada (NSERC), École de technology supérieure (ÉTS) within the LASSENA Laboratory in collaboration with two industrial partners namely iMetrik Global Inc. and Future Electronics.

\section{REFERENCES}

N. Alam, A. T. Balaei, and A. G. Dempster, "A DSRC Doppler-based cooperative positioning enhancement for vehicular networks with GPS availability," Vehicular Technology, IEEE Transactions on, vol. 60, pp. 4462-4470, 2011.

[2] G. Taylor, J. Li, D. Kidner, C. Brunsdon, and M. Ware, "Modelling and prediction of GPS availability with digital photogrammetry and LiDAR," International Journal of Geographical Information Science, vol. 21, pp. 1-20, 2007.

[3] W. Zongyuan, L. Huanzhang, Z. Shuanghong, and S. Feng, "The study of square root cubature Kalman smoother and its application on INS/GPS integrated navigation," in Mechatronics and Automation (ICMA), 2014 IEEE International Conference on, 2014, pp. 1827-1832.

[4] L. Zhao, H. Qiu, and Y. Feng, "Study of Robust Filtering Application in Loosely Coupled INS/GPS System," Mathematical Problems in Engineering, vol. 2014, 2014.

[5] M. S. Grewal, A. P. Andrews, and C. G. Bartone, Global Navigation Satellite Systems, Inertial Navigation, and Integration: John Wiley \& Sons, 2013.

[6] P. D. Groves, Principles of GNSS, inertial, and multisensor integrated navigation systems: Artech House, 2013.

[7] D.-J. Jwo and F.-I. Chang, "A fuzzy adaptive fading Kalman filter for GPS navigation," in Advanced Intelligent Computing Theories and 
Applications. With Aspects of Theoretical and Methodological Issues, ed: Springer, 2007, pp. 820-831.

[8] M. R. Taha, A. Noureldin, and N. El-Sheimy, "Improving INS/GPS positioning accuracy during GPS outages using fuzzy logic," in Proceedings of the 16th International Technical Meeting of the Satellite Division of The Institute of Navigation (ION GPS/GNSS 2003), 2001, pp. 499-508.

[9] Q. Xia, M. Rao, Y. Ying, and X. Shen, "Adaptive fading Kalman filter with an application," Automatica, vol. 30, pp. 1333-1338, 1994.

[10] K. H. Kim, J. G. Lee, and C. G. Park, "Adaptive two-stage EKF for INS-GPS loosely coupled system with unknown fault bias," Positioning, vol. $1,2006$.

[11] G. T. Schmidt and R. E. Phillips, "INS/GPS integration architectures," DTIC Document2010.

[12] G. Falco, G. A. Einicke, J. T. Malos, and F. Dovis, "Performance analysis of constrained loosely coupled GPS/INS integration solutions," Sensors, vol. 12, pp. 15983-16007, 2012.

[13] J. G. Ryan and D. M. Bevly, "On the Observability of Loosely Coupled Global Positioning System/Inertial Navigation System Integrations With Five Degree of Freedom and Four Degree of Freedom Inertial Measurement Units," Journal of Dynamic Systems, Measurement, and Control, vol. 136, p. 021023, 2014.

[14] I. Skog and P. Handel, "Time synchronization errors in loosely coupled GPS-aided inertial navigation systems," Intelligent Transportation Systems, IEEE Transactions on, vol. 12, pp. 10141023, 2011.

[15] Y. Bar-Shalom, X. R. Li, and T. Kirubarajan, Estimation with applications to tracking and navigation: theory algorithms and software: John Wiley \& Sons, 2004.

[16] J. Škaloud, "Optimizing georeferencing of airborne survey systems by INS/DGPS," Citeseer, 1999.

[17] D. Bhatt, P. Aggarwal, V. Devabhaktuni, and P. Bhattacharya, "A new source difference artificial neural network for enhanced positioning accuracy," Measurement Science and Technology, vol. 23, p. 105101, 2012.

[18] L. M. Bergasa, J. Nuevo, M. A. Sotelo, R. Barea, and M. E. Lopez, "Real-time system for monitoring driver vigilance," Intelligent Transportation Systems, IEEE Transactions on, vol. 7, pp. 63-77, 2006.

[19] A. Noureldin, T. B. Karamat, M. D. Eberts, and A. El-Shafie, "Performance enhancement of MEMSbased INS/GPS integration for low-cost navigation applications," Vehicular Technology, IEEE Transactions on, vol. 58, pp. 1077-1096, 2009.

[20] D. Loebis, R. Sutton, J. Chudley, and W. Naeem, "Adaptive tuning of a Kalman filter via fuzzy logic for an intelligent AUV navigation system," Control engineering practice, vol. 12, pp. 1531-1539, 2004.

[21] H. Ying, Y. Ding, S. Li, and S. Shao, "Comparison of necessary conditions for typical Takagi-Sugeno and Mamdani fuzzy systems as universal approximators," Systems, Man and Cybernetics, Part A: Systems and Humans, IEEE Transactions on, vol. 29, pp. 508-514, 1999.

[22] A. Hamam and N. D. Georganas, "A comparison of Mamdani and Sugeno fuzzy inference systems for evaluating the quality of experience of HaptoAudio-Visual applications," in Haptic Audio visual Environments and Games, 2008. HAVE 2008. IEEE International Workshop on, 2008, pp. 87-92.

[23] K. Belarbi, F. Titel, W. Bourebia, and K. Benmahammed, "Design of Mamdani fuzzy logic controllers with rule base minimisation using genetic algorithm," Engineering Applications of Artificial Intelligence, vol. 18, pp. 875-880, 10// 2005.

[24] T. J. Ross, Fuzzy logic with engineering applications: John Wiley \& Sons, 2009.

[25] D. Simon, "Training fuzzy systems with the extended Kalman filter," Fuzzy Sets and Systems, vol. 132, pp. 189-199, 2002.

[26] D.-J. Jwo and T.-S. Cho, "A practical note on evaluating Kalman filter performance optimality and degradation," Applied Mathematics and Computation, vol. 193, pp. 482-505, 2007.

[27] R. Vershynin, "How close is the sample covariance matrix to the actual covariance matrix?," Journal of Theoretical Probability, vol. 25, pp. 655-686, 2012. 